\section{Recomendaciones y conductas para el manejo anestésico de pacientes sometidos a cirugía cardíaca pediátrica durante la pandemia del SARS-CoV-2}

\author{
Recommendations and actions for the anesthetic management of \\ pediatric cardiac surgery during the SARS-CoV-2 pandemic
}

Dra. Lourdes Carolina Pellecer-González, ${ }^{*}$ Dr. Orlando Tamariz-Cruz*,‡

RESUMEN. La pandemia generada por el nuevo coronavirus SARS-CoV-2 ha marcado la historia de la humanidad de manera indeleble. Las conductas, acciones y hábitos de las personas han cambiado sustancialmente redefiniendo nuestras actividades en todos los niveles. Nuestra especialidad está dedicada a la atención de pacientes pediátricos con cardiopatías congénitas, desde etapa neonatal hasta la adolescencia. En este grupo de edad el escenario sintomático denominado COVID-19 no está presente en la mayoría de los casos, por lo que la práctica anestésica se realiza con precauciones dirigidas para atender pacientes asintomáticos considerados no portadores del virus SARS-CoV-2 y sin los elementos completos de los equipos de protección personal. Este documento compila las prácticas y lineamientos adoptados por los anestesiólogos del programa KARDIAS para la atención de niños con cardiopatías congénitas durante la pandemia por COVID-19. Hemos realizado una adaptación de las diferentes guías reportadas del manejo anestésico, pediátrico, cardiovascular y ecocardiográfico, apegándonos a la evidencia reportada hasta la fecha. Estas recomendaciones son aplicables para cualquier centro que realice anestesia cardiovascular pediátrica con modificaciones según sus requerimientos institucionales, y deberán ser revisadas periódicamente considerando la nueva evidencia científica reportada. Finalmente, comentamos sobre la aparición de una probable relación clínica de infección por SARS-CoV2 y una variedad atípica de enfermedad de Kawasaki en niños.

ABSTRACT. The pandemic produced by the new SARS-CoV-2 coronavirus has indelibly marked the history of humanity. The conducts, actions and habits of people have changed substantially, redefining our activities at all levels. Our specialty is dedicated to the care of pediatric patients with congenital heart defects, from the neonatal stage to adolescence. In this age group, the symptomatic picture of COVID-19 is not present in most cases, therefore, anesthetic practice is carried out with precautions aimed to assist asymptomatic patients considered not carriers of the virus SARS-CoV-2, without the complete elements of personal protective equipment. This document compiles the practices and guidelines adopted by the anesthetists of the KARDIAS program for the care of children with congenital heart defects during the COVID-19 pandemic. We have made an adaptation of the different guidelines reported on anesthetic, pediatric, cardiovascular and echocardiographic management, adhering to the evidence reported to date. These recommendations are applicable for any center that performs pediatric cardiovascular anesthesia with modifications according to its institutional requirements and they should be periodically reviewed regarding new scientific evidence reported. Finally, we comment about the appearance of a probable new clinical presentation of SARS-CoV-2 infection as an atypical variety of Kawasaki disease in children.

\section{INTRODUCCIÓN}

D iferentes sociedades nacionales e internacionales de diferentes especialidades médicas han publicado guías y recomendaciones para conducirse médicamente durante la pandemia COVID-19; sin embargo, no hay ninguna específica para la práctica de la anestesia cardiovascular pediátrica, por lo que hemos realizado una revisión de las diferentes publicaciones del manejo anestésico, pediátrico, cardiovascular y ecocardiográfico basados en la evidencia reportada hasta la fecha.
Anestesiología

Octubre-Diciembre 2020

Vol. 43. No. 4. pp 230-240

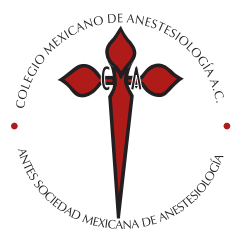

Palabras clave:

SARS-CoV-2, COVID-19, cardiopatías congénitas.

Keywords:

SARS-CoV-2, COVID-19, congenital heart defects.

* Centro Pediátrico del Corazón ABC-KARDIAS, Fundación KARDIAS A.C. México.

‡ División de Cirugía Cardiovascular, Instituto Nacional de Pediatría, Secretaría de Salud. México.

Solicitud de sobretiros: Dra. Lourdes Carolina Pellecer-González Calle Dr. Márquez Núm. 76, Apt. 308A, Col. Doctores, 06720, Alcaldía Cuauhtémoc, Ciudad de México, México. E-mail: lpellecer@hotmail.com

Recibido para publicación: 12-05-2020

Aceptado para publicación: 14-07-2020 
La anestesia cardiovascular pediátrica colabora con todas las disciplinas relacionadas con el cuidado del paciente quirúrgico con cardiopatía congénita, participa desde la selección de los pacientes en las sesiones médico-quirúrgicas hasta el manejo postoperatorio. En este documento han sido consideradas las directrices que el anestesiólogo cardiovascular pediátrico debe observar durante cada actividad realizada, en consenso con las otras disciplinas, durante la epidemia generada por el virus SARS-CoV-2 y su manifestación clínica COVID-19, tanto en el Centro Pediátrico del Corazón ABC-Kardias como en el Instituto Nacional de Pediatría en la Ciudad de México.

Estas recomendaciones se encuentran sujetas a cambios y deberán ser revisadas periódicamente ante la nueva evidencia científica o políticas nacionales (o Internacionales) para el control de la epidemia.

\section{DEFINICIONES Y RECOMENDACIONES}

Definir adecuadamente la condición de cada paciente nos permitirá tomar decisiones en cuanto a la realización de pruebas diagnósticas para infección por SARS-CoV-2 y el uso apropiado de los equipos de protección personal (EPP).

La fuerza de trabajo para definición del adecuado empleo de los EPP del Hospital de Niños de Texas (Texas Children's Hospital) en Estados Unidos emitió recomendaciones considerando una situación de escasez nacional sobre el empleo de los EPP, y la realización de pruebas diagnósticas al atender pacientes programados a cirugías cardíacas pediátricas de emergencia o urgentes; es recomendable adaptar estas definiciones a las condiciones institucionales de cada centro.

Definiciones para pacientes que serán intervenidos para corrección de cardiopatías congénitas a quienes se realizan pruebas diagnósticas para SARS-CoV-2 (PD CoV-2):

- Paciente en vigilancia de COVID-19: prueba diagnóstica en curso en un paciente asintomático sin factor epidemiológico de riesgo.

- Paciente en investigación de COVID-19: prueba diagnóstica en curso en un paciente con factor(es) epidemiológico(s) de riesgo y/o cuadro clínico compatible con COVID-19.

- Paciente positivo para COVID-19: paciente con prueba diagnóstica positiva para SARS-CoV-2.

\section{RECOMENDACIONES EN RELACIÓN CON EPP Y PRUEBAS DIAGNÓSTICAS}

1. Paciente con PD CoV-2 negativa: se recomienda uso de EPP adecuado en todo el personal expuesto a procedimientos generadores de aerosol, aun si no presenta síntomas o factores epidemiológicos de riesgo.
2. Paciente en vigilancia o investigación de COVID-19: se recomienda uso de EPP adecuado en todo el personal expuesto a procedimientos generadores de aerosol, cuando no se conozca el resultado de la PD CoV-2.

3. Procedimientos quirúrgicos: es recomendado realizar la PD CoV-2 (RT-PCR) en todos los pacientes que se presenten para cirugía cardiovascular pediátrica. La prueba debe ser realizada el día previo a la cirugía, en pacientes electivos o en el momento en que se presente, si es cirugía de emergencia o urgencia. Se recomienda el uso de EPP adecuado a todo el personal expuesto a procedimientos generadores de aerosol.

4. Unidad de Cuidados Intensivos Cardiovasculares (UCICV): se recomienda realizar la PD CoV-2 en todos los pacientes con cardiopatía congénita que ingresen a la UCICV. Se recomienda el empleo de EPP adecuados por todo el personal expuesto a procedimientos generadores de aerosol.

5. Pacientes neonatos con factores epidemiológicos de riesgo: se recomienda uso de EPP adecuado en la atención del parto o cesárea de embarazadas en investigación o positivas de COVID-19, y con diagnóstico prenatal de cardiopatía congénita en el producto; así como la realización de PD CoV-2 al neonato (Tabla 1).

\section{PRIORIZACIÓN DE LOS PROCEDIMIENTOS QUIRÚRGICOS}

Las autoridades en salud de México han determinado que en los hospitales que no son centro de concentración para pacientes COVID-19 sólo deben intervenirse pacientes con emergencia o urgencia quirúrgica. Recientemente han sido publicadas recomendaciones de las diferentes cardiopatías congénitas con prioridad quirúrgica por grupos de edad. El programa KARDIAS se basa en estas indicaciones para regular los procedimientos quirúrgicos con prioridad decreciente en tres niveles: emergencia, urgencia o electivo de alta prioridad (Tabla 2).

\section{RECOMENDACIONES PARA ECOCARDIOGRAFÍA}

La realización de ecocardiografía transesofágica (ETE) debe ser pospuesta o cancelada si no es estrictamente necesario. El personal que lo realiza debe ser limitado, así como el tiempo del estudio, llevándose a cabo con un protocolo preestablecido que permita realizarlo buscando las vistas cardíacas necesarias en el menor tiempo posible.

No está claro cuál es el papel de la sonda de ETE en la diseminación de aerosol por su colocación o manipulación, pero debido a que en el contexto de cirugía cardíaca pediátrica la sonda de ETE es colocada inmediatamente después de la intubación, debe ser considerada como potencial generadora de aerosoles. 
Tabla 1: Equipo de protección personal recomendado por actividad y rol asignado.

\begin{tabular}{|c|c|c|c|c|}
\hline Tipo de paciente & Lugar & Personal & Actividad & EPP \\
\hline \multirow{6}{*}{$\begin{array}{l}\text { En vigilancia } \\
\text { Paciente asintomático y sin } \\
\text { exposición de riesgo }\end{array}$} & \multirow[t]{2}{*}{$\begin{array}{l}\text { Consulta/Evaluación/ } \\
\text { Encamamiento }\end{array}$} & $\begin{array}{l}\text { Paciente y } \\
\text { acompañante }\end{array}$ & Cualquier actividad & Mascarilla quirúrgica \\
\hline & & Personal sanitario & Cualquier actividad & $\begin{array}{l}\text { Mascarilla quirúrgica } \\
\text { Bata } \\
\text { Guantes } \\
\text { Protección ocular }\end{array}$ \\
\hline & \multirow[t]{2}{*}{$\begin{array}{l}\text { Quirófano o Terapia } \\
\text { Intensiva }\end{array}$} & Paciente & $\begin{array}{l}\text { Previo a manipulación de la vía } \\
\text { aérea }\end{array}$ & Mascarilla quirúrgica \\
\hline & & Personal sanitario & $\begin{array}{l}\text { Manipulación de vía aérea: } \\
\text { entubación, recolocación de TOT, } \\
\text { ETE, extubación, broncoscopía, } \\
\text { otros }\end{array}$ & $\begin{array}{l}\text { Doble gorro } \\
\text { Respirador N95 } \\
\text { Protección ocular } \\
\text { Bata } \\
\text { Doble guante } \\
\text { Botas }\end{array}$ \\
\hline & \multirow[t]{2}{*}{$\begin{array}{l}\text { Quirófano o Terapia } \\
\text { Intensiva }\end{array}$} & Paciente & $\begin{array}{l}\text { Cualquier actividad que no } \\
\text { manipule la vía aérea }\end{array}$ & Mascarilla quirúrgica \\
\hline & & Personal sanitario & $\begin{array}{l}\text { Cualquier actividad que no } \\
\text { manipule la vía aérea }\end{array}$ & $\begin{array}{l}\text { Doble gorro } \\
\text { Respirador N95 } \\
\text { Protección ocular } \\
\text { Bata } \\
\text { Doble guante } \\
\text { Botas }\end{array}$ \\
\hline \multirow{6}{*}{$\begin{array}{l}\text { En investigación } \\
\text { Paciente sintomático y/o con } \\
\text { exposición de riesgo } \\
0 \\
\text { Caso positivo }\end{array}$} & \multirow[t]{2}{*}{$\begin{array}{l}\text { Consulta/Evaluación/ } \\
\text { Encamamiento }\end{array}$} & $\begin{array}{l}\text { Paciente y } \\
\text { acompañante }\end{array}$ & Cualquier actividad & Mascarilla N95 \\
\hline & & Personal sanitario & Cualquier actividad & $\begin{array}{l}\text { Doble gorro } \\
\text { Respirador N95 } \\
\text { Protección ocular } \\
\text { Bata } \\
\text { Doble guante }\end{array}$ \\
\hline & \multirow[t]{2}{*}{$\begin{array}{l}\text { Quirófano o Terapia } \\
\text { Intensiva }\end{array}$} & Paciente & $\begin{array}{l}\text { Previo a manipulación de la vía } \\
\text { aérea }\end{array}$ & Mascarilla N95 \\
\hline & & Personal sanitario & $\begin{array}{l}\text { Manipulación de vía aérea: } \\
\text { entubación, recolocación de TOT, } \\
\text { ETE, extubación, broncoscopía, } \\
\text { otros }\end{array}$ & $\begin{array}{l}\text { Doble gorro } \\
\text { Respirador N95 } \\
\text { Protección ocular } \\
\text { Bata } \\
\text { Doble guante } \\
\text { Botas }\end{array}$ \\
\hline & \multirow[t]{2}{*}{$\begin{array}{l}\text { Quirófano o Terapia } \\
\text { Intensiva }\end{array}$} & Paciente & $\begin{array}{l}\text { Cualquier actividad que no } \\
\text { manipule la vía aérea }\end{array}$ & Mascarilla N95 \\
\hline & & Personal sanitario & $\begin{array}{l}\text { Cualquier actividad que no } \\
\text { manipule la vía aérea }\end{array}$ & $\begin{array}{l}\text { Doble gorro } \\
\text { Respirador N95 } \\
\text { Protección ocular } \\
\text { Bata } \\
\text { Doble guante } \\
\text { Botas }\end{array}$ \\
\hline
\end{tabular}

Modificado de: OMS "Rational use of personal protective equipment for coronavirus disease 2019" 2020. CDC "Interim Infection Prevention and Control Recommendations for Patients with Suspected or Confirmed Coronavirus Disease 2019 in Healthcare Settings" 2020 Abreviaturas: EPP: equipos de protección personal, TOT: tubo orotraqueal, ETE: ecocardiograma transesofágico. 
Tabla 2: Priorización de procedimientos quirúrgicos.

\begin{tabular}{|c|c|c|c|}
\hline Neonatos & $\begin{array}{l}\text { Emergente (cirugía dentro } \\
\text { de las primeras } 24-48 \\
\text { horas del diagnóstico con } \\
\text { recursos adecuados) }\end{array}$ & $\begin{array}{l}\text { Urgente (cirugía dentro de } \\
\text { las primeras } 1-2 \text { semanas } \\
\text { del diagnóstico con } \\
\text { recursos adecuados) }\end{array}$ & $\begin{array}{l}\text { Electiva de alta prioridad } \\
\text { (cirugía después de dos } \\
\text { semanas del diagnóstico } \\
\text { con adecuados recursos)* }\end{array}$ \\
\hline \multicolumn{4}{|l|}{ Cortocircuitos } \\
\hline CATVP/cor triatriatum & \multirow[t]{3}{*}{ Obstruida } & \multirow{3}{*}{$\begin{array}{l}\text { Gradiente que incrementa } \\
<1 \text { semana con septum íntegro }\end{array}$} & \\
\hline TGA & & & 2-4 semanas con CIV \\
\hline Tronco arterioso & & & Si está estable \\
\hline Tetralogía de Fallot & Hipoxemia severa/crisis de hipoxia & Sintomático & \\
\hline \multicolumn{4}{|c|}{ Lesiones regurgitantes (insuficiencias) } \\
\hline Anomalía de Ebstein & & \multicolumn{2}{|l|}{ Refractario a tratamiento médico } \\
\hline \multicolumn{4}{|l|}{ Lesiones obstructivas } \\
\hline Coartación aórtica & Choque persistente a pesar de PGE & \multicolumn{2}{|l|}{ Si se logra estabilizar con PGE } \\
\hline Coartación aórtica crítica & Choque persistente a pesar de PGE & \multicolumn{2}{|l|}{ Si se logra estabilizar con PGE } \\
\hline \multicolumn{4}{|c|}{ Flujo pulmonar dependiente de PGE } \\
\hline $\begin{array}{l}\text { Atresia pulmonar con } \\
\text { septum íntegro }\end{array}$ & & \multicolumn{2}{|l|}{ Si no se cuenta con stents } \\
\hline \multicolumn{4}{|c|}{ Flujo sistémico dependiente de PGE } \\
\hline Ventrículo izquierdo hipoplásico & $\begin{array}{l}\text { Septum íntegro o restrictivo si no se } \\
\text { cuenta con septostomía con balón }\end{array}$ & $\begin{array}{l}\text { Decisión del cirujano } \\
\text { de acuerdo al caso }\end{array}$ & $\begin{array}{l}\text { Decisión del cirujano de } \\
\text { acuerdo al caso }\end{array}$ \\
\hline \multicolumn{4}{|l|}{ Otras } \\
\hline Fístulas & Trombosis de la fístula & \multicolumn{2}{|l|}{ Estenosis de la fístula } \\
\hline Arritmias & \multicolumn{3}{|c|}{ Bloqueo AV completo congénito que no es posible manejar médicamente o con marcapasos externo } \\
\hline ALCAPA & \multicolumn{3}{|c|}{ Una vez estabilizado médicamente } \\
\hline
\end{tabular}

\section{Lactantes}

\section{Cortocircuitos de izquierda a derecha CIV \\ Cortocircuitos de derecha a izquierda Tetralogía de Fallot \\ Lesiones regurgitantes \\ Defecto septal atrioventricular}

Anomalía de Ebstein

Insuficiencia mitral

Insuficiencia aórtica

Lesiones obstructivas

Prótesis valvulares

Estenosis aórtica/lesión obstructiva del VI

Obstrucción del tracto de salida del VD

\section{Otras}

Fístulas

Cardiomiopatía dilatada/ insuficiencia cardíaca

Candidato a Glenn bidireccional
ICC sintomática con manejo médico

Sintomático (crisis de hipoxia, cianosis) con manejo médico

Aguda y hemodinámicamente inestable

Válvula trombosada

Trombosis de la fístula

Estenosis de la fístula
Falla en la mejoría (progresión) de la ICC

Sintomático (crisis de hipoxia, cianosis) con manejo médico

Pacientes con síndrome de Down y sobrecirculación, considerar la edad del paciente para la reparación, insuficiencia significativa no controlada con tx. médico

IC derecha progresiva a pesar del tratamiento médico

ICC sintomática con tratamiento médico

Crecimiento ventricular izquierdo, < de la FEVI, sintomático

\section{Reducción de la FEVI, síntomas}

Reducción de la función del VD ICC con falla a tx. médico
Falla en la mejoría (progresión) de la ICC Incremento en la cianosis en paciente con fístula, estenosis de la fístula 
Continuación de la Tabla 2: Priorización de procedimientos quirúrgicos.

\begin{tabular}{|c|c|c|c|}
\hline Pediátricos & $\begin{array}{l}\text { Emergente (cirugía dentro } \\
\text { de las primeras } 24-48 \\
\text { horas del diagnóstico con } \\
\text { recursos adecuados) }\end{array}$ & $\begin{array}{l}\text { Urgente (cirugía dentro de } \\
\text { las primeras } 1-2 \text { semanas } \\
\text { del diagnóstico con } \\
\text { recursos adecuados)* }\end{array}$ & $\begin{array}{l}\text { Electiva de alta prioridad } \\
\text { (cirugía después de dos } \\
\text { semanas del diagnóstico con } \\
\text { adecuados recursos)* }\end{array}$ \\
\hline \multicolumn{4}{|l|}{ Lesiones regurgitantes } \\
\hline Insuficiencia mitral & & & $\begin{array}{l}\text { ICC sintomática con } \\
\text { tratamiento médico }\end{array}$ \\
\hline Insuficiencia aórtica & $\begin{array}{l}\text { Aguda y hemodinámicamente } \\
\text { inestable }\end{array}$ & & $\begin{array}{l}\text { Crecimiento de VI, disminución } \\
\text { de la función, síntomas }\end{array}$ \\
\hline \multicolumn{4}{|l|}{ Lesiones obstructivas } \\
\hline $\begin{array}{l}\text { Estenosis aórtica/obstrucción } \\
\text { del tracto de salida del VI }\end{array}$ & & & $\begin{array}{l}\text { Disminución de la función } \\
\text { del VI, síntomas }\end{array}$ \\
\hline Prótesis valvulares & Prótesis trombosada & & \\
\hline Fístula VD a arteria pulmonar & $\begin{array}{l}\text { Estenosis severa con disfunción } \\
\text { del VD y/o arritmias ventriculares }\end{array}$ & $\begin{array}{l}\text { Estenosis severa con } \\
\text { disfunción del VD y/o presión } \\
\text { del VD igual a la sistémica }\end{array}$ & Empeoramiento de falla derecha \\
\hline \multicolumn{4}{|l|}{ Otras } \\
\hline $\begin{array}{r}\text { Cardiomiopatía dilatada/ } \\
\text { insuficiencia cardíaca }\end{array}$ & & ICC con falla en el tx. médico & $\begin{array}{l}\text { Falla en la mejoría } \\
\text { (progresión) de la ICC }\end{array}$ \\
\hline Candidato a Fontan & & & Cianosis en incremento \\
\hline Endocarditis & $\begin{array}{l}\text { Falla en la mejoría } \\
\text { (progresión) de la ICC }\end{array}$ & $\begin{array}{l}\text { Hemodinámicamente estable, } \\
\text { pero con infección incontrolable }\end{array}$ & Orientarse por guías de manejo \\
\hline $\begin{array}{l}\text { Origen anómalo de } \\
\text { las coronarias }\end{array}$ & $\begin{array}{l}\text { Falla de la mejoría } \\
\text { (progresión) de la ICC }\end{array}$ & $\begin{array}{l}\text { Historia de muerte súbita } \\
\text { (abortada), dolor torácico } \\
\text { de bajo esfuerzo }\end{array}$ & \\
\hline $\begin{array}{l}\text { Lesiones combinadas ( } \mathrm{p} . \text { ej.: } \\
\text { IM y estenosis subaórtica) }\end{array}$ & $\begin{array}{l}\text { Falla en la mejoría } \\
\text { (progresión) de la ICC }\end{array}$ & $\begin{array}{l}\text { Lesiones individuales } \\
\text { moderadas a severas }\end{array}$ & \\
\hline
\end{tabular}

* Nota: El tiempo de diagnóstico para las categorías depende de los recursos disponibles, protocolos institucionales y otros casos pendientes

ALCAPA = arteria coronaria izquierda anómala desde la arteria pulmonar, $\mathrm{AV}=$ aurículo-ventricular, $\mathrm{CIV}=$ comunicación interventricular, $\mathrm{FEVI}=$ fracción de expulsión del ventrículo

izquierdo, ICC = insuficiencia cardíaca congestiva, $\mathrm{PGE}=$ prostaglandina $\mathrm{E}, \mathrm{TX}=$ tratamiento, $\mathrm{VD}=$ ventrículo derecho, $\mathrm{VI}=$ ventrículo izquierdo.

Modificado de: Stephens EH, Dearani JA, Guleserian KJ, et al. COVID-19: crisis management in congenital heart surgery. Ann Thorac Surg. 2020.

La Sociedad Americana de Ecocardiografía ha emitido recomendaciones en cuanto al tipo de protección del personal que realizará el ecocardiograma, definiendo protección estándar, protección contra gotas o protección contra aerosoles.

- Equipo y protección estándar: lavado o higiene de manos y uso de guantes desechables cuando el estudio sea realizado en pacientes en vigilancia de COVID-19.

- Protección contra gotas o aerosoles: uso de bata impermeable, guantes desechables, botas (si es en el quirófano), cubrebocas quirúrgico, respirador y protección ocular. Esto aplica para todos los pacientes en vigilancia o confirmados para COVID-19 dentro o fuera de quirófano.

Los EPP están relacionados con el tipo de procedimientos ecocardiográficos, transtorácicos o transesofágicos, y con el sitio de realización (laboratorio de ecocardiografía, hospitalización, urgencia, hemodinamia, unidad de terapia intensiva o quirófano) como se especifica en las definiciones (Figura 1).

El equipo de ecocardiografía e inclusive la sonda de ETE serán protegidos con plástico o cobertores que permitan su desinfección adecuada posterior a la realización del estudio. La sonda de ETE debe seguir un proceso de lavado y desinfección en un contenedor adecuado y de acuerdo con las indicaciones del proveedor y normas hospitalarias bioinfecciosas.

Las consideraciones en relación con el empleo de los EPP, la protección contra secreciones y desinfección de la sonda de ETE, especialmente en pacientes quirúrgicos, son mostradas en las figuras 1 a 3.

\section{RECOMENDACIONES PARA EL MANEJO DE LA VÍA AÉREA}

En todos los pacientes en vigilancia o confirmados de infección por el virus SARS-CoV-2 tanto la intubación como la extubación deben ser realizadas considerando una planificación y justificación precisa.

El procedimiento debe ser llevado a cabo en una habitación individual cerrada y de preferencia con presión negativa. El personal sanitario será limitado a tres personas, dos médicos expertos en el manejo de la vía aérea y una enfermera circulante, todos con equipo de protección personal colocado adecua- 


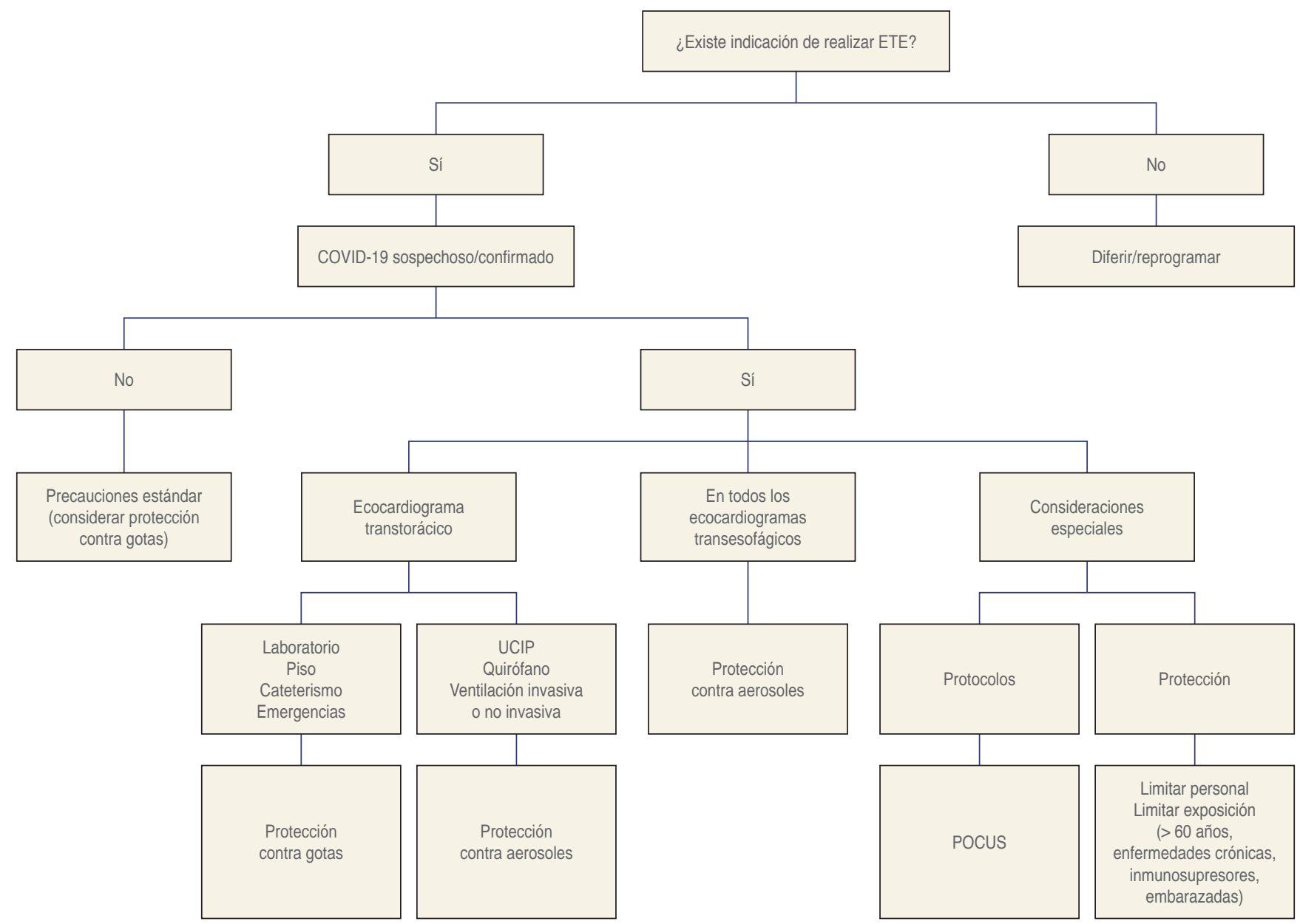

Figura 1: Algoritmo para determinar el nivel de protección del personal que realiza el estudio de ecocardiografía.

Abreviaturas: ETE = ecocardiografía transesofágica, UCIP = Unidad de Terapia Intensiva Pediátrica, POCEC: $=$ ecografía en el punto de atención.

Modificado de: Kirkpatrick JN, Mitchell C, Taub C, Kort S, Hung J, Swaminathan M. ASE Statement on

Protection of Patients and Echocardiography Service Providers During the 2019 Novel Coronavirus

Outbreak: Endorsed by the American College of Cardiology. J Am Coll Cardiol. 2020;75:3078-3084 .

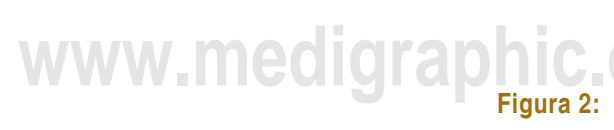

Equipo de desinfección de sonda de ecocardiografía transesofágica. De no contar con el equipo diseñado por el proveedor (como el mostrado en la imagen), utilizar contenedores individuales con jabón enzimático, ortoftaldehido al $0.55 \%\left(\right.$ CIDEX $^{*}$ OPA $\left.{ }^{\circledR}\right)$ y solución

salina. Sumergir la sonda durante 10 minutos en jabón enzimático, enjuagar; sumergir la sonda en ortoftaldehido durante 5 minutos, enjuagar; sumergir la sonda durante 10 minutos en solución

salina. Dejar secar al aire. Fuente: Colección del autor.

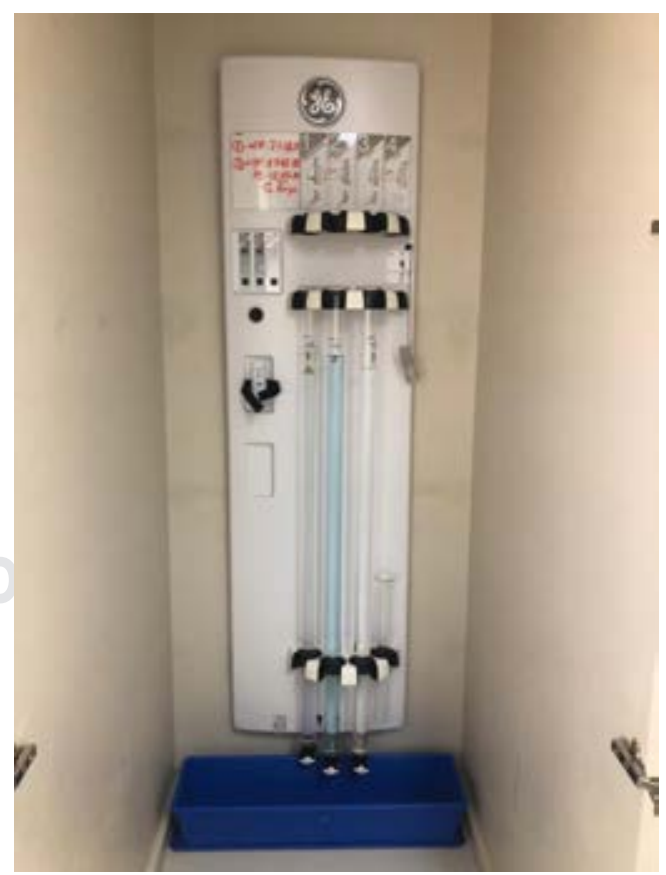



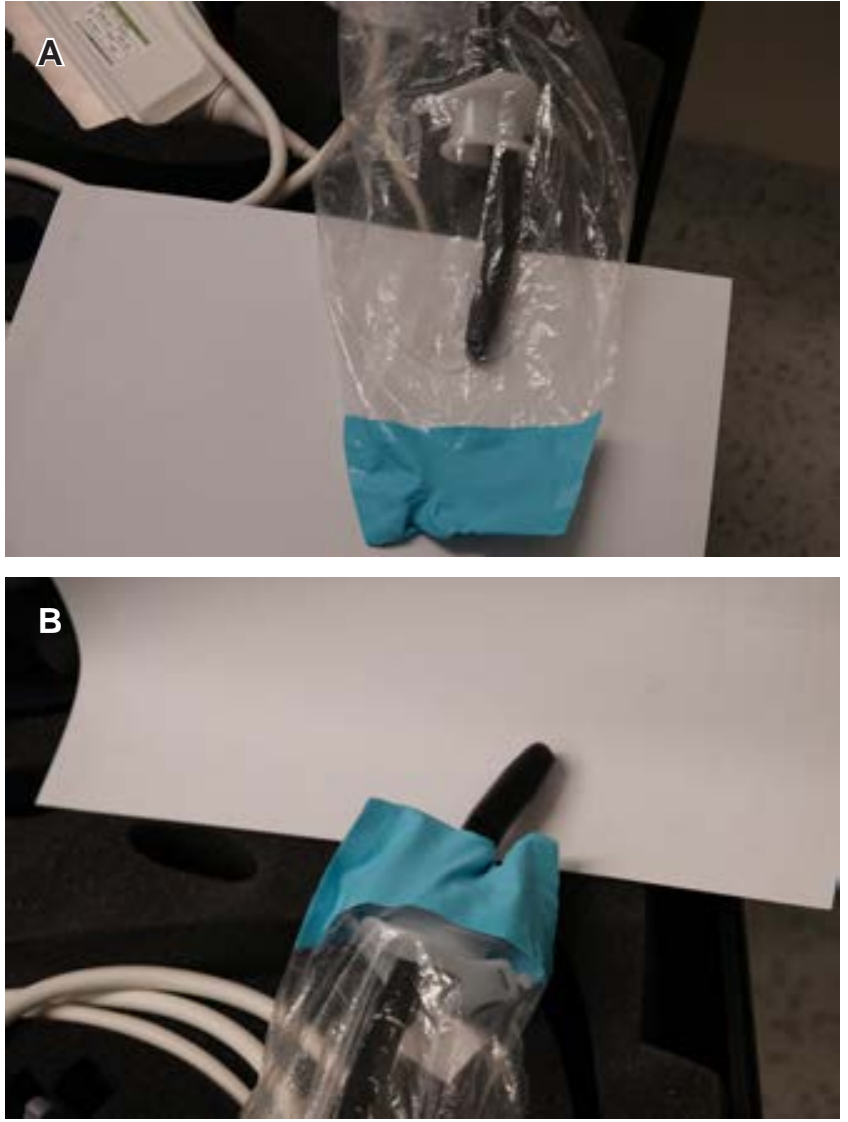

Figura 3: Cobertor para la sonda del ETE. A) Introducir la boquilla dentro del cobertor plástico, orientar hacia la parte distal del cobertor el lado que sujetará el paciente con los incisivos. Posteriormente introducir la sonda del ETE a través de la boquilla como se muestra en la imagen. B. Colocar la boquilla en los incisivos del paciente y posteriormente introducir la sonda de ETE de la manera habitual. Al retirar la sonda, el cobertor plástico impedirá la diseminación de aerosoles o gotas. Fuente: Colección del autor. Abreviaturas: ETE: ecocardiograma transesofágico.

Fuente: Colección del autor.

damente. No será permitido el ingreso o egreso del personal cuando sea realizado el procedimiento y serán considerados los recambios de aire por hora del sistema de ventilación de la habitación o quirófano para calcular el tiempo en el que puede salir o ingresar personal después de realizada la intubación o extubación (Tabla 3).

El uso de la caja acrílica como método de barrera para los aerosoles no se adapta a la práctica de los pacientes pediátricos con cardiopatías congénitas, ya que al tener pacientes neonatos, lactantes o pediátricos a quienes se les colocará un acceso venoso central superior y sonda de ETE se hace necesaria la remoción de este dispositivo posterior a la intubación. Como alternativa utilizamos una bolsa plástica transparente, la cual se puede manipular sin omitir la barrera de la vía aérea (Figura 4).

Las simulaciones son altamente recomendadas para todo el personal sanitario que se encuentre relacionado con el
Tabla 3: Tiempo requerido para eliminación de contaminantes en relación con los recambios de aire por hora.

\begin{tabular}{ccc}
$\begin{array}{c}\text { Cambios } \\
\text { de aire } \\
\text { por hora }\end{array}$ & $\begin{array}{c}\text { Tiempo requerido } \\
\text { para remover } \\
\text { eficientemente } \\
\text { el } 99 \% \\
\text { (minutos) }\end{array}$ & $\begin{array}{c}\text { Tiempo requerido } \\
\text { para remover } \\
\text { eficientemente } \\
\text { el 99.9\% } \\
\text { (minutos) }\end{array}$ \\
\hline 2 & 138 & 207 \\
4 & 69 & 104 \\
6 & 46 & 69 \\
8 & 35 & 52 \\
10 & 28 & 41 \\
12 & 23 & 35 \\
15 & 18 & 28 \\
20 & 14 & 21 \\
50 & 6 & 8 \\
\hline
\end{tabular}

Modificado de: Centers of Disease Control and Prevention. Guidelines for environmental infection control in health-care facilities. 2003.

manejo de la vía aérea para disminuir errores que produzcan contaminación.

También es recomendado el uso de listas de verificación para corroborar la adecuada implementación de los pasos críticos: colocación del EPP, planificación y asignación de roles, equipo, medicamentos y material necesario; previo a la intubación/extubación, después de la intubación/extubación y retiro de EPP. El adecuado retiro de EPP y manejo de desechos reutilizables y no reutilizables deben ser considerados integralmente como parte final del manejo de la vía aérea.

\section{COLOCACIÓN Y RETIRO DE EPP}

Todo el personal sanitario debe estar familiarizado con los protocolos de la institución con relación a la colocación y retiro de EPP. Esto se realizará en parejas y con una lista de chequeo para poder asegurar su práctica adecuada. Se debe prestar mayor atención al retiro del EPP, ya que es donde el personal se encuentra más expuesto a contaminación (Tabla 4).

\section{ANÁLISIS}

Compartimos los lineamientos que empleamos en el programa KARDIAS (Centro Pediátrico del Corazón ABC-Kardias e Instituto Nacional de Pediatría) para el manejo de pacientes en quienes será realizada la cirugía cardíaca pediátrica. Estas recomendaciones han sido diseñadas con base en revisiones realizadas por sociedades nacionales e internacionales de anestesiología, pediatría, anestesia cardiovascular, ecocardiografía, Organización Mundial de la Salud (OMS), Centro para el Control de Enfermedades (CDC) y la Secretaría de Salud de México. 
Tabla 4: Listas de verificación en pasos críticos.

Planeación

Asignación de roles

$\square$ Médico especialista en vía aérea 1, líder, intuba

$\square$ Médico especialista en vía aérea 2, asistencia

$\square$ Enfermero circulante, dentro de la sala

$\square$ Enfermero corredor, fuera de la sala

\section{Previsión}

$\square$ Medicamentos, monitoreo y tiempo

$\square$ ¿Cómo contactar ayuda?

$\square$ Equipo extra necesario

$\square$ Desecho de materiales

$\square$ Cuidados del equipo

$\square$ Descontaminación del equipo usado en la vía aérea

\section{Vía aérea difícil no prevista}

(Dos intentos fallidos de intubación por personal entrenado en el manejo de la vía aérea)

$\square$ ¿Si se presenta VAD no prevista, se despertará al paciente y se reprogramará el procedimiento?

Plan para el manejo de VAD no prevista

$\square$ A: Intubación endotraqueal por médico experto en el manejo de la vía aérea

$\square$ B: Mascarilla laríngea

$\square$ C: Cricotomía de urgencia

$\square$ Todos los miembros del equipo confirman estar de acuerdo con el plan

Intubación

\section{Extubación}

\section{Equipo}

$\square$ Máquina de anestesia/ventilador probados

$\square$ Monitor básico de signos vitales con capnografía

$\square$ Caja acrílica

$\square$ Succión funcionando

$\square$ Videolaringoscopio

$\square$ Laringoscopio y hojas

$\square$ Mascarilla laríngea

$\square$ Equipo de traqueostomía de urgencia

$\square$ Pinza de TOT

$\square$ Máquina de ultrasonido

$\square$ Sonda de ETE

$\square$ Boquilla

\section{Material}

$\square$ Un circuito de anestesia/ventilación

$\square$ Un circuito Bain con filtro HEPA

$\square$ Dos mascarillas faciales acojinadas

$\square$ Dos filtros HEPA

$\square$ Trampa y línea de capnografía

$\square$ Dos cánulas orofaríngeas

$\square$ Una cánula Yankauer

$\square$ Un estilete

$\square$ Tres tubos orotraqueales

$\square$ Cinta y jeringa de globo

$\square$ Gasas y parches oculares

$\square$ Una bolsa para desechos reutilizables

$\square$ Un campo plástico transparente grande

$\square$ Dos campos impermeables

\section{Medicamentos}

$\square$ Peso del paciente

$\square$ Alergias conocidas

$\square$ Opioide

$\square$ Hipnótico

$\square$ Relajante neuromuscular

$\square$ Vasopresor

$\square$ Drogas de emergencia

$\square$ Sedación/analgesia de mantenimiento
Equipo

$\square$ Máquina de anestesia/ventilador, misma que usa

$\square$ Monitor básico de signos vitales con capnografía, mismo que usa

$\square$ Caja acrílica

$\square$ Succión funcionando

$\square$ Videolaringoscopio

$\square$ Laringoscopio y hojas

$\square$ Mascarilla laríngea

$\square$ Equipo de traqueostomía de urgencia

$\square$ Pinza de TOT

\section{Material}

$\square$ Un circuito de anestesia/ventilación, mismo que usa

$\square$ Un circuito Bain con filtro HEPA

$\square$ Una mascarilla facial acojinada

$\square$ Dos filtros HEPA, mismos que usa

$\square$ Trampa y línea de capnografía, mismos que usa

$\square$ Dos cánulas orofaríngeas

$\square$ Una cánula Yankauer

$\square$ Un estilete

$\square$ Tres tubos orotraqueales

$\square$ Cinta y jeringa de globo

$\square$ Gasas y parches oculares

$\square$ Una bolsa para desechos reutilizables

$\square$ Un campo plástico transparente grande

$\square$ Dos campos impermeables

$\square$ Dispositivo de flujo de aire con el que continuará (puntas nasales, mascarilla facial reservorio, etc.)

$\square$ Mascarilla quirúrgica

\section{Medicamentos}

$\square$ Peso del paciente

$\square$ Alergias conocidas

$\square$ Sedación/analgesia de mantenimiento

$\square$ Verificar uso de antiemético previo al procedimiento

$\square$ Opioide

$\square$ Hipnótico

$\square$ Relajante neuromuscular

$\square$ Vasopresor

$\square$ Drogas de emergencia 
Continuación de la Tabla 4: Listas de verificación en pasos críticos.

Intubación

Extubación

Verificar antes del procedimiento

Verificar antes del procedimiento

\section{Ventilación}

$\square$ Máquina de anestesia o ventilador probado

\section{Monitoreo colocado y funcionando}

$\square$ Capnografía

$\square \mathrm{SpO}_{2}$

$\square \mathrm{ECG}$

$\square$ PANI

Medicamentos, equipo y material en la mesa

$\square$ Circuito Bain con filtro HEPA

$\square$ Laringoscopio con hojas

$\square$ Dos mascarillas laríngeas

$\square$ Tres tubos orotraqueales

$\square$ Dos cánulas orofaríngeas

$\square$ Estilete

$\square$ Bolsa para desecho de material reutilizable

$\square$ Opioide

$\square$ Hipnótico

$\square$ Relajante neuromuscular

$\square$ Vasopresor y drogas de emergencia

$\square$ Sedación/analgesia de mantenimiento

\section{Verificar}

$\square$ Accesos IV

$\square$ Optimizar posición (rampa, Trendelenburg reverso, etc.)

$\square$ Optimizar condición del paciente antes de la inducción (fluidos, vasopresor, inotrópicos, etc.)

\section{Equipo y material en la cama del paciente}

$\square$ Dos campos impermeables

$\square$ Una bolsa plástica transparente grande o caja de acrílico

$\square$ Circuito de anestesia/ventilación con mascarilla facial acojinada y doble filtro

\section{HEPA}

$\square$ Una cánula orofaríngea

$\square$ Cánula Yankauer con succión funcionando

$\square$ Videolaringoscopio con hoja

$\square$ Parches oculares

$\square$ Gasas

$\square$ Cinta para fijar

$\square$ Pinza para TOT

$\square$ Boquilla para ETE

\section{Ventilación}

$\square$ Máquina de anestesia o ventilador, misma que usa

Monitoreo colocado y funcionando, mismo que usa

$\square$ Capnografía

$\square \mathrm{SpO}_{2}$

$\square \mathrm{ECG}$

$\square$ PANI

Medicamentos, equipo y material en la mesa

$\square$ Circuito Bain con filtro HEPA

$\square$ Laringoscopio con hojas

$\square$ Dos mascarillas laríngeas

$\square$ Tres tubos orotraqueales

$\square$ Dos cánulas orofaríngeas

$\square$ Estilete

$\square$ Videolaringoscopio con hoja

$\square$ Parches oculares

$\square$ Gasas

$\square$ Cinta para fijar

$\square$ Bolsa para desecho de material reutilizable

$\square$ Opioide

$\square$ Hipnótico

$\square$ Relajante neuromuscular

$\square$ Vasopresor y drogas de emergencia

$\square$ Sedación/analgesia de mantenimiento

\section{Verificar}

$\square$ Accesos IV

$\square$ Administración previa de antieméticos

$\square$ Optimizar posición (rampa, Trendelenburg reverso, etc.)

$\square$ Optimizar condición del paciente antes de la extubación (nebulización, aspiración de secreciones, etc.)

Equipo y material en la cama del paciente

$\square$ Dos campos impermeables

$\square$ Una bolsa plástica transparente grande o caja de acrílico

$\square$ Mascarilla facial acojinada

$\square$ Una cánula orofaríngea

$\square$ Cánula Yankauer con succión funcionando

$\square$ Pinza para TOT

$\square$ Dispositivo de flujo de aire con el que continuará (puntas nasales, mascarilla facial reservorio, etc.)

$\square$ Mascarilla quirúrgica

\section{Verificar después del procedimiento}

\section{$\square$ Fijación y profundidad del TOT}

$\square$ Ventilación adecuada

$\square$ Mantenimiento de sedación y analgesia

$\square$ Colocar adecuadamente los desechos en la bolsa apropiada

$\square$ Colocar adecuadamente el equipo reutilizable en la bolsa apropiada

$\square$ Tiempo adecuado para salida/ingreso de quirófano

\section{Verificar después del procedimiento}
$\square$ Ventilación adecuada
$\square$ Suministro de oxígeno adecuado
$\square$ Estabilidad hemodinámica
$\square$ Optimizar la sedoanalgesia del paciente
$\square$ Colocar adecuadamente los desechos en la bolsa apropiada
$\square$ Colocar adecuadamente el equipo reutilizable en la bolsa apropiada
$\square$ Tiempo adecuado para salida/ingreso de quirófano

Fuente: Colección del autor.

Abreviaturas: VAD: vía aérea difícil, TOT: tubo orotraqueal, ETE: ecocardiograma transesofágico, HEPA: filtro de partículas de aire de alta eficiencia, SPO2: pulso oxímetro de pulso, EKG: electrocardiograma, PANI: presión arterial no invasiva. 

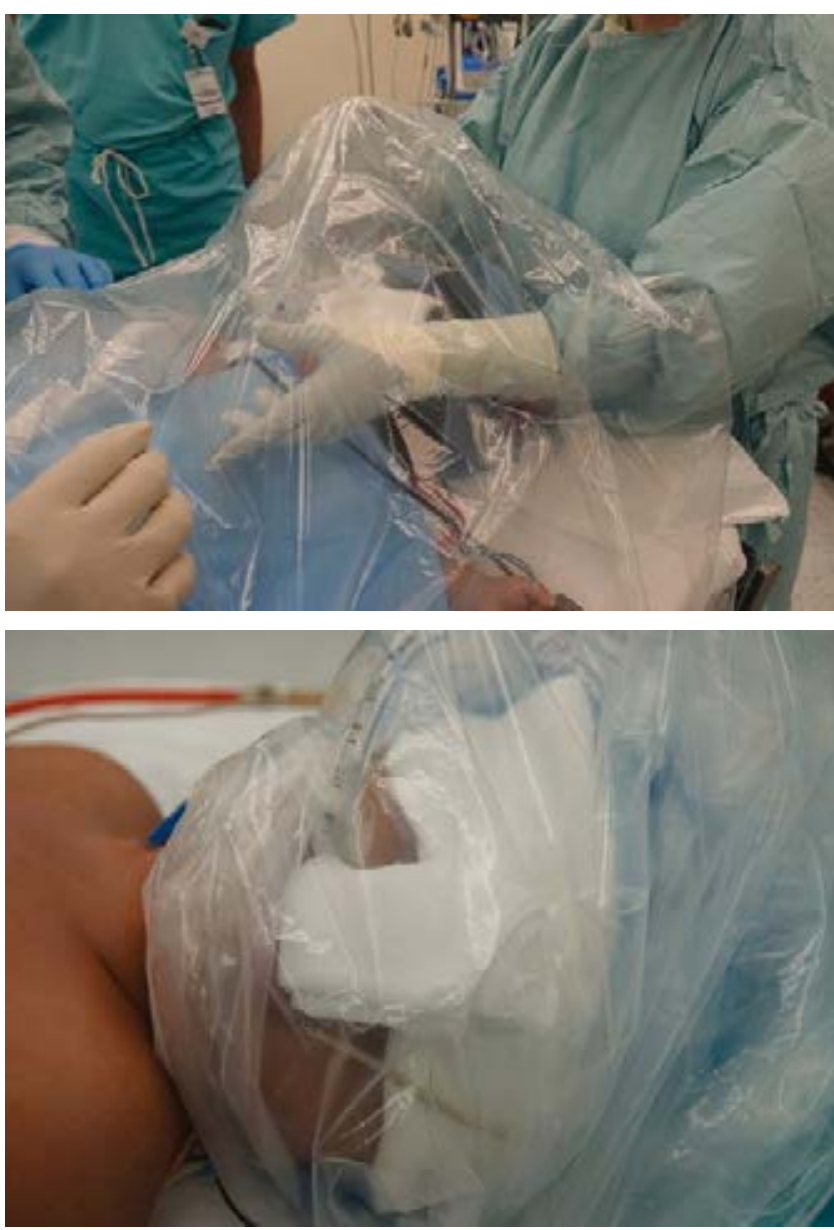

Figura 4: Bolsa plástica como método de barrera para aerosoles. El uso de la caja acrílica en pacientes pediátricos puede obstaculizar la colocación de accesos venosos centrales superiores o la preparación del área quirúrgica del tórax. El uso de un plástico transparente brinda una alternativa efectiva como aislante de barrera flexible para la manipulación en los pacientes pediátricos.

Fuente: Colección del autor.

La mayor parte de la atención en los medios, y por ende de la bibliografía, ha sido enfocada en la población adulta en vista de la severidad del cuadro clínico; sin embargo, la población pediátrica no ha tenido tanta consideración en vista de que cuando se presenta el cuadro respiratorio es leve en su mayoría, llegando incluso a no tener síntomas. Debido a esto, se considera que los pacientes con cardiopatías congénitas que necesitan cirugía de emergencia o urgencia podrían ser potenciales transmisores asintomáticos de la infección al personal de salud, por lo que se recomiendan las condiciones de seguridad adecuadas.
Desde el año 2005 se reportó la asociación entre un «nuevo» tipo de coronavirus relacionado con el SARS-CoV y la enfermedad de Kawasaki. El virus fue identificado como $\mathrm{H}-\mathrm{CoV}-\mathrm{NH}$ o coronavirus de New Haven y se alertó ante la posibilidad de que el contagio de niños con dilatación aneurismática de las coronarias, característica de la enfermedad de Kawasaki, fuera por vía aérea y relacionado con «nuevas cepas» de coronavirus humano $(\mathrm{H}-\mathrm{CoV})$. Recientemente, un grupo de médicos en Inglaterra ha descrito un cuadro clínico de especial interés en la población infantil que sugiere sospecha de COVID-19, y está caracterizado como una forma atípica de enfermedad de Kawasaki y choque tóxico. En estos pacientes hay síntomas abdominales inespecíficos como dolor, distensión y/o diarrea, hallazgos sanguíneos como linfopenia e incremento en los niveles de proteína $C$ reactiva. En algunos niños con esas manifestaciones clínicas se han obtenido pruebas de RT-PCR positivas para SARS-CoV-2, pero aún no se cuenta con la evidencia necesaria para establecer una relación directa entre la infección de este virus y la enfermedad de Kawasaki, por lo que sería importante continuar con la investigación en esta línea.

Si bien las observaciones descritas son preliminares, su reporte debe conducir al incremento de las medidas de protección del personal de salud ante la posibilidad de atender cuadros atípicos de COVID-19 y resalta la importancia de no considerar únicamente las manifestaciones respiratorias cuando realizamos manejo de la vía aérea en niños en consecuencia de intervenciones quirúrgicas de cualquier especialidad. Lo más preocupante en lo que se refiere a la seguridad del personal de salud, es que en la mayoría de las instituciones no se proporciona el EPP para la atención de niños sin síntomas respiratorios. Consideramos que este concepto es erróneo y deber ser modificado.

Varios hospitales pediátricos de México han sido convertidos en centros híbridos con áreas destinadas para pacientes con COVID-19 y otras para urgencias médico-quirúrgicas no relacionadas con COVID-19; es así que hospitales como el Instituto Nacional de Pediatría y el Centro Pediátrico del Corazón ABC, ambos parte del programa KARDIAS, se han convertido en un ambiente desafiante para los pacientes que requieren cirugía cardíaca pediátrica de emergencia o urgencia al ser tratados en centros donde no se encuentran exentos del riesgo de contagio.

Es imperativa la necesidad de contar con protocolos bien fundamentados para actuar con eficacia y seguridad en la práctica de la anestesia cardiovascular pediátrica en tiempos de la pandemia COVID-19. 


\section{LECTURAS RECOMENDADAS}

1. Rothe C, Schunk M, Sothmann P, et al. Transmission of 2019-nCoV infection from an asymptomatic contact in Germany. N Engl J Med. 2020;382:970-971.

2. Bai Y, Yao L, Wei T, et al. Presumed asymptomatic carrier transmission of COVID-19. JAMA. 2020;323:1406-1407.

3. Li R, Pei S, Chen B, et al. Substantial undocumented infection facilitates the rapid dissemination of novel coronavirus (SARS-CoV-2). Science. 2020;368:489-493.

4. Hoehl S, Rabenau H, Berger A, et al. Evidence of SARS-CoV-2 infection in returning travelers from Wuhan, China. N Engl J Med. 2020;382:12781280.

5. Ng OT, Marimuthu K, Chia PY, et al. SARS-CoV-2 infection among travelers returning from Wuhan, China. N Engl J Med. 2020;382:14761478. doi: 10.1056/NEJMc2003100.

6. Main COVID-19: https://www.cdc.gov/coronavirus/2019-nCoV/index. html / Individuals at high risk: https:/www.cdc.gov/coronavirus/2019ncov/specific-groups/high-risk- complications.html.

7. Children and COVID-19. Disponible en: https://www.cdc.gov/ coronavirus/2019-ncov/faq.html\#anchor 1584387482747.

8. Pregnancy and COVID-19. Disponible en: https://www.cdc.gov/ coronavirus/2019-ncov/prepare/pregnancy- breastfeeding.html

9. Guidance from the White House, the U.S. Department of Health and Human Services (HHS), and CDC. Disponible en: https://www. coronavirus.gov/

10. World Health Organization COVID-19 site. Disponible en: https://www. who.int/emergencies/diseases/novel-coronavirus-2019

11. AAP Healthy Children COVID-19 site. Disponible en: https://www. healthychildren.org/English/health-issues/conditions/chest- lungs/ Pages/2019-Novel-Coronavirus.aspx

12. Adult Congenital Heart Association webinar on March 16, 2020 on Coronavirus and the CHD patient. Disponible en: https://youtu.be/ hou2qM1phVQ

13. Sisters By Heart Facebook Live event from March 17, 2020 on Coronavirus and Children with CHD. Disponible en: https://www. facebook.com/156971814350396/videos/2817193955063709/
14. COVID-19 information from Conquering CHD. Disponible en: https:// www.conqueringchd.org/covid-19-update/

15. ASE Statement on Protection of Patients and Echocardiography Service Providers During the 2019 Novel Coronavirus Outbreak. ASEcho.org

16. Xiaoxia L, Zhang L, Du H. SARS-CoV-2 infection in children. NEJM. 2020. doi: 10.1056/NEJMc2005073.

17. Zhao S, Ling K, Yan H, Zhong L, Peng X, Yao S, et al. Anesthetic Management of patients with suspected or confirmed 2019 Novel coronavirus infection during emergency procedures. J Cardiovasc Vasc Anesth. 2020. Disponible en: https://doi.org/10.1053/j.jvca.2020.02.039

18. Zucco L, Levy N, Ketchandji D, Aziz M, Ramachandran SK. Recommendations for airway management in patient with suspected coronavirus (2019-nCoV) Infection. www.apsf.org

19. Zimmermann P, Curtis N. Coronavirus infections in children including COVID-19. An overview of the epidemiology, clinical features, diagnosis, treatment and prevention options in children. Pediatr Infect Dis J. 2020. doi: 10.1097/INF.0000000000002660.

20. American College of Surgeons. COVID-19: Considerations for optimum surgical protection before, during and after operation. www.facs.org

21. Esper F, Shapiro ED, Weibel C, Ferguson D, Landry ML, Kahan JS. Association between a novel human coronavirus and Kawasaki disease. JID. 2005;191:499.502.

22. North Central London CCG's Incident Coordination Centre. North Central London Clinical Commissioning Group. Significant alert in respect of Children and Pediatric shock for urgent action. 2020.

23. Rowley AH, Shulman ST. The epidemiology and pathogenesis of Kawasaki disease. Front Pediatr. 2018;6:374. doi: 10.3389/ fped.2018.00374.

24. Riphagen S, Gomez X, González-Martínez C. Hiperinflammatory shock in children during COVID-19 pandemic. Lancet. 2020. Disponible en: doi.org/10.1016/S0140-6736(20)31094-1

25. Pellecer L y Tamariz-Cruz O. Definiciones operativas y protocolo anestésico durante la pandemia producida por SARS-CoV-2 en un programa de cirugía cardíaca pediátrica. Revista Chilena de Anestesia 2020; 49: 372-387 https://doi.org/10.25237/revchilanestv49n03.016 